Letter

\title{
A systemic whole-plant change in redox levels accompanies the rapid systemic response of Arabidopsis to wounding
}

\section{Yosef Fichman and Ron Mittler*}

The Division of Plant Sciences and Interdisciplinary Plant Group, College of Agriculture, Food and Natural Resources, and The Department of Surgery, University of Missouri School of Medicine. Christopher S. Bond Life Sciences Center University of Missouri. 1201 Rollins St, Columbia, MO 65201.

*Corresponding author: $\underline{\text { mittlerr@missouri.edu }}$

\section{Running title}

Systemic redox wave response to wounding

\section{One sentence summary}

The wounding-induced reactive oxygen species (ROS) wave is accompanied by a systemic whole-plant redox response.

\begin{abstract}
Reactive oxygen species (ROS) play a key role in regulating plant responses to different abiotic stresses, wounding and pathogen attack. In addition to triggering responses at the tissues directly subjected to stress, ROS were recently shown to mediate a rapid whole-plant systemic signal, termed the "ROS wave", required for inducing a state of systemic acquired acclimation, or systemic wound response. However, whether the ROS wave that spreads from the local tissues subjected to wounding to the rest of the plant triggers alterations in redox levels, is mostly unknown at present. Here, using a genetically-encoded reporter for cellular glutathione redox changes, roGFP1, we show that the wounding-induced systemic ROS wave in Arabidopsis thaliana is accompanied by a rapid systemic wave of cytosolic redox oxidation, termed a "redox wave". The ROS wave may therefore trigger changes in redox levels in systemic leaves that in turn can trigger transcriptional, metabolic and proteomic changes resulting in acclimation and/or systemic wound responses.
\end{abstract}




\section{Introduction}

Reactive oxygen species (ROS) play a central role in the regulation of plant responses to different developmental signals, abiotic stresses, wounding and pathogen attack (e.g., Chang et al., 2004; Fryer et al., 2004; Mhamdi and Van Breusegem, 2018; Kollist et al., 2019). One of the major routes by which ROS cause an alteration in gene expression in response to many of these stimuli is a change in the redox state of different proteins (Mittler, 2017; Huang et al., 2019). Such proteins could include transcriptional regulators, phosphatases, kinases and different pumps and channels that link ROS signaling to a variety of different cellular responses and control plant acclimation and defense (Mittler, 2017; Huang et al., 2019). In addition to triggering responses at the tissues directly subjected to abiotic stress, ROS were recently shown to regulate rapid whole-plant systemic responses that occur at tissues not directly subjected to the stress, inducing a state of systemic acquired acclimation (SAA), or systemic wound response (SWR) (e.g., Miller et al., 2009; Szechyńska-Hebda et al., 2010; Suzuki et al., 2013; Gilroy et al., 2016; Fichman et al., 2019; Zandalinas et al., 2019; Zandalinas et al., 2020a). The ROS-dependent systemic signaling pathway mediating these processes, termed "the ROS wave" (Miller et al., 2009), was recently shown to be regulated by respiratory burst oxidase homolog proteins (RBOHD and RBOHF) and to be mediated through the vascular bundles of plants (Zandalinas et al., 2020b). However, whether the ROS wave that spreads from the local tissues subjected to stress to the rest of the plant triggers alterations in redox levels, is mostly unknown at present.

\section{Results and Discussion}

The development of genetically-encoded reporters for cellular and glutathione redox changes, and their application in plants led to major advancements in the study of redox and ROS signaling in recent years (e.g., Schwarzländer et al., 2008; Meyer and Dick, 2010; Rosenwasser et al., 2010; Schwarzländer et al., 2016; Exposito-Rodriguez et al., 2017; Lim et al., 2019; Nietzel et al., 2019; García-Quirós et al., 2020; Haber and Rosenwasser, 2020). These reporters have however been primarily used in conjunction with confocal microscopy, limiting their application to the detection of redox changes in specific cells, tissues and organs. In contrast, whole-plant detection of redox changes in mature plants grown in soil has been limited. We recently developed a method for live whole-plant imaging of ROS levels in soil-grown plants and used it to study the ROS wave in wild type plants and different mutants responding to different stimuli (e.g., Devireddy et al., 2020; Fichman et al., 2020; Zandalinas et al., 2020a). Although changes in ROS levels (detected as accumulation of oxidized DCF in cells; Fichman et al., 2019) were demonstrated to accompany the systemic response of plants to different stimuli and these changes were shown to result in metabolic and transcriptomic changes that drove SAA or SWR (e.g., Suzuki et al., 2013; Zandalinas et al., 2019; Zandalinas et al., 2020a), it is not known whether a systemic whole-plant redox response also accompanies this rapid signaling process. To test this possibility, we studied cytosolic roGFP1-expressing plants (Jiang et al., 2006; Meyer et al., 2007; Schwarzländer et al., 2008; Supplementary material Methods) subjected to a local injury stimuli (Figures 1 and 2). The choice of roGFP1 was based on its highly reduced state at the cytosol which provides a low background in unstressed plants, and the choice of wounding was a result of not wanting to impact the roGFP1 sensor by treatments such as heat or high light stress that may alter its activity. As shown in Figure $1 \mathrm{~A}$, wounding of a single leaf of a wild type (Col-0) plant, grown in soil, resulted in the triggering of a systemic ROS wave response, imaged by the accumulation of oxidized DCF fumigated as $\mathrm{H}_{2}$ DCFDA into whole-plants (Fichman et al., 2019). As shown in Figure $1 B$ and 
Supplementary Movie 1, wounding of a single leaf of a cytosolic-expressing roGFP1 transgenic plant (Jiang et al., 2006), grown in soil, resulted in local and systemic changes in the redox state of the roGFP1 probe, evident by raw changes in Excitation/Emission at $420 \mathrm{~nm} / 520 \mathrm{~nm}$ (for oxidized roGFP1) and Excitation/Emission of $480 \mathrm{~nm} / 520 \mathrm{~nm}$ (for reduced roGFP1) fluorescence. To quantify these changes and to calculate degree of oxidized roGFP1 in local and systemic tissues in response to wounding, we next determined the levels of $420 \mathrm{~nm} / 520 \mathrm{~nm}$ (for oxidized roGFP1) and $480 \mathrm{~nm} / 520 \mathrm{~nm}$ (for reduced roGFP1) fluorescence in whole plants fumigated with $5 \mathrm{mM}$ hydrogen peroxide $\left(\mathrm{H}_{2} \mathrm{O}_{2}\right.$; to induce roGFP1 oxidation), or $5 \mathrm{mM}$ Dithiothreitol (DTT; to induce roGFP1 reduction), for $15 \mathrm{~min}$, and calculated the ratio of 420/480 $\mathrm{nm}$ for these treatments (Figure 2A). Using these ratios we then determined the $420 / 480 \mathrm{~nm}$ ratio and degree of roGFP1 oxidation in local and systemic leaves of the control and wounded plants shown in Figure 1 (Figure 2B and Supplementary Movies 2 and 3). The results shown in Figures 1B, 2 and Supplementary Movies 1-3, reveal that wounding of a single Arabidopsis leaf results in the oxidation of the cytosolic roGFP1 probe in both local and systemic leaves, and that these changes correspond to the changes recorded with the $\mathrm{H}_{2}$ DCFDA probe (Figure $1 \mathrm{~A}$ ). Similar degrees of roGFP oxidation were recently reported for whole-plant imaging of chloroplastic roGFP2-expressing plants subjected to light stress (Haber and Rosenwasser, 2020), further supporting our findings and demonstrating that the degree of increase in roGFP oxidation in whole plants upon enhanced ROS accumulation is in the range of 5-10\% (Figure 1B).

Our findings that wounding of a single leaf is accompanied by a systemic wave of ROS production (Fichman et al., 2019; Figure 1A), as well as a change in cytosolic redox levels (a "redox wave"; Figures 1B, 2; Supplementary Movies 1-3) could therefore provide an initial clue to how the systemic ROS wave response alters the levels of metabolites and transcripts in systemic tissues causing an enhanced state of SAA or SWR (Miller et al., 2009; Szechyńska-Hebda et al., 2010; Suzuki et al., 2013; Gilroy et al., 2016; Fichman et al., 2019; Zandalinas et al., 2019; Zandalinas et al., 2020a, 2020b). Interestingly, the roGFP1 probe remained oxidized for at least 60 min following wounding, showing that the ROS wave response is turned "on" for at least 1 hour following wounding (Figure 1B). This finding is in agreement with our recent findings that the systemic ROS wave response to excess light stress remains "on" for at least 3 hours following the initial application of the $10 \mathrm{~min}$ high light stress treatment to the local leaf (Devireddy et al., 2020). Further studies are of course needed to identify the different regulatory proteins altered by the ROS and redox waves in response to a local stimuli and to tie their altered activity to the systemic response they cause. One potential regulator, recently identified during systemic responses to light stress, is MYB30 (Fichman et al., 2020). Because changes in MYB30 protein oxidation were shown to alter its DNA binding activity, MYB30 could be a potential regulator that links changes in redox levels in systemic tissues to transcript expression and SAA (Tavares et al., 2014; Fichman et al., 2020). In addition to demonstrating that the ROS wave is accompanied by a change in redox levels in local and systemic tissues (Figures 1 and 2; Supplementary Movies 1-3), our study also demonstrates that at least some genetically-encoded reporters can be imaged in whole-plants grown in soil, using a sufficiently sensitive apparatus (Fichman et al., 2019; Figures 1, 2, and Supplementary Movies 1-3). This finding opens the way for further studies of ROS and redox signaling in whole plants grown in soil, and perhaps even to large-scale phenotyping studies. 


\section{Acknowledgments}

The roGFP1 seeds were obtained from the Arabidopsis Biological Resource Center (ABRC). This work was supported by funding from the National Science Foundation (MCB-1936590, IOS-1932639, and IOS1353886), and the University of Missouri. We apologize to all authors of papers not mentioned in this manuscript due to space limitations.

\section{References}

Chang CCC, Ball L, Fryer MJ, Baker NR, Karpinski S, Mullineaux PM (2004) Induction of Ascorbate Peroxidase 2 expression in wounded Arabidopsis leaves does not involve known wound-signalling pathways but is associated with changes in photosynthesis. Plant J 38: 499-511

Devireddy AR, Liscum E, Mittler R (2020) Phytochrome B is required for systemic stomatal responses and ROS signaling during light stress in Arabidopsis. Plant Physiol doi.org/10.1104/pp.20.01084

Exposito-Rodriguez M, Laissue PP, Yvon-Durocher G, Smirnoff N, Mullineaux PM (2017) Photosynthesis-dependent $\mathrm{H} 2 \mathrm{O} 2$ transfer from chloroplasts to nuclei provides a high-light signalling mechanism. Nat Commun 8: 49

Fichman Y, Miller G, Mittler R (2019) Whole-plant live imaging of reactive oxygen species. Mol Plant 12: 1203-1210

Fichman Y, Zandalinas SI, Sengupta S, Burks D, Myers RJ, Azad R, Mittler R (2020) MYB30 orchestrates systemic reactive oxygen signaling and plant acclimation. Plant Physiol doi: 10.1104/pp.20.00859

Fryer MJ, Ball L, Oxborough K, Karpinski S, Mullineaux PM, Baker NR (2003) Control of Ascorbate Peroxidase 2 expression by hydrogen peroxide and leaf water status during excess light stress reveals a functional organisation of Arabidopsis leaves. Plant J 33: 691-705

García-Quirós E, Alché JDD, Karpinska B, Foyer CH, Wilson Z (2020) Glutathione redox state plays a key role in flower development and pollen vigour. J Exp Bot 71: 730-741

Gilroy S, Białasek M, Suzuki N, Górecka M, Devireddy AR, Karpiński S, Mittler R (2016) ROS, calcium, and electric signals: Key mediators of rapid systemic signaling in plants. Plant Physiol 171: 16061615

Haber Z, Rosenwasser S (2020) Resolving the dynamics of photosynthetically produced ROS by highresolution monitoring of chloroplastic $\mathrm{E}_{\mathrm{GSH}}$ in Arabidopsis. bioRxiv 2020.03.04.976092

Huang J, Willems P, Wei B, Tian C, Ferreira RB, Bodra N, Martínez Gache SA, Wahni K, Liu K, Vertommen D, et al (2019) Mining for protein S-sulfenylation in Arabidopsis uncovers redoxsensitive sites. Proc Natl Acad Sci U S A 116: 21256-21261

Jiang K, Schwarzer C, Lally E, Zhang S, Ruzin S, Machen T, Remington SJ, Feldman L (2006) Expression and characterization of a redox-sensing green fluorescent protein (reduction-oxidation-sensitive green fluorescent protein) in Arabidopsis. Plant Physiol 141: 397-403

Kollist H, Zandalinas SI, Sengupta S, Nuhkat M, Kangasjärvi J, Mittler R (2019) Rapid responses to abiotic stress: priming the landscape for the signal transduction network. Trends Plant Sci 24: 2537 
Lim SD, Kim S-H, Gilroy S, Cushman JC, Choi W-G (2019) Quantitative ROS bioreporters: a robust toolkit for studying biological roles of ROS in response to abiotic and biotic stresses. Physiol Plant 165: 356-368

Meyer AJ, Brach T, Marty L, Kreye S, Rouhier N, Jacquot JP, Hell R (2007) Redox-sensitive GFP in Arabidopsis thaliana is a quantitative biosensor for the redox potential of the cellular glutathione redox buffer. Plant J 52: 973-986

Meyer AJ, Dick TP (2010) Fluorescent protein-based redox probes. Antioxidants Redox Signal 13: 621650

Mhamdi A, Van Breusegem F (2018) Reactive oxygen species in plant development. Dev 145: $\operatorname{dev} 164376$

Miller G, Schlauch K, Tam R, Cortes D, Torres MA, Shulaev V, Dangl JL, Mittler R. (2009) The plant NADPH oxidase RBOHD mediates rapid systemic signaling in response to diverse stimuli. Sci Signal. 2: ra45

Mittler R (2017) ROS are good. Trends Plant Sci 22: 11-19

Nietzel T, Elsässer M, Ruberti C, Steinbeck J, Ugalde JM, Fuchs P, Wagner S, Ostermann L, Moseler A, Lemke $P$, et al (2019) The fluorescent protein sensor roGFP2-Orp1 monitors in vivo $\mathrm{H} 2 \mathrm{O} 2$ and thiol redox integration and elucidates intracellular $\mathrm{H} 2 \mathrm{O} 2$ dynamics during elicitor-induced oxidative burst in Arabidopsis. New Phytol 221: 1649-1664

Rosenwasser S, Rot I, Meyer AJ, Feldman L, Jiang K, Friedman H (2010) A fluorometer-based method for monitoring oxidation of redox-sensitive GFP (roGFP) during development and extended dark stress. Physiol Plant 138: 493-502

Schwarzländer M, Dick TP, Meyer AJ, Morgan B (2016) Dissecting redox biology using fluorescent protein sensors. Antioxidants Redox Signal 117: 13810-13820

Schwarzländer M, Fricker MD, Müller C, Marty L, Brach T, Novak J, Sweetlove U, Hell R, Meyer AJ (2008) Confocal imaging of glutathione redox potential in living plant cells. J Microsc 231: 299-316

Suzuki N, Miller G, Salazar C, Mondal HA, Shulaev E, Cortes DF, Shuman JL, Luo X, Shah J, Schlauch K, et al (2013) Temporal-spatial interaction between reactive oxygen species and abscisic acid regulates rapid systemic acclimation in plants. Plant Cell 25: 3553-3569

Szechyńska-Hebda M, Kruk J, Górecka M, Karpińska B, Karpiński S (2010) Evidence for light wavelength-specific photoelectrophysiological signaling and memory of excess light episodes in Arabidopsis. Plant Cell 22: 2201-2218

Tavares CP, Vernal J, Delena RA, Lamattina L, Cassia R, Terenzi H. (2014) S-nitrosylation influences the structure and DNA binding activity of AtMYB30 transcription factor from Arabidopsis thaliana. Biochim Biophys Acta. 1844: 810-817

Zandalinas SI, Fichman Y, Devireddy AR, Sengupta S, Azad RK, Mittler R (2020a) Systemic signaling during abiotic stress combination in plants. Proc Natl Acad Sci U S A 117: 13810-13820

Zandalinas SI, Fichman Y, Mittler R (2020b) Vascular bundles mediate systemic reactive oxygen signaling during light stress in Arabidopsis. Plant Cell doi.org/10.1105/tpc.20.00453

Zandalinas SI, Sengupta S, Burks D, Azad RK, Mittler R (2019) Identification and characterization of a 
core set of ROS wave-associated transcripts involved in the systemic acquired acclimation response of Arabidopsis to excess light. Plant J 98: 126-141

Figure Legends:

\section{Figure 1. Imaging of ROS and redox levels in whole plants subjected to wounding}

A. Representative time-lapse images of whole-plant ROS levels (indicated by DCF oxidation) in wild type Arabidopsis thaliana (Col-0) plants, untreated (control, top), or wounded (wounding, bottom; applied to leaf $L$ only), are shown on left; representative line graphs showing continuous measurements of ROS levels in local (L) and systemic (S) leaves over the entire course of the experiment ( 0 to $60 \mathrm{~min}$ ) are shown at the middle (ROls used to generate the line graphs are indicated with dashed yellow ovals on left); and statistical analysis of ROS levels in local and systemic leaves at 0 and $60 \mathrm{~min}$ is shown on right.

B. Same as in A., but for transgenic plants overexpressing the roGFP1 protein at the cytosol (Jiang et al., 2006). Fluorescence was measured at excitation/emission (ex/em) of $420 \mathrm{~nm} / 520 \mathrm{~nm}$ for oxidized roGFP1 (middle, in green; oxidized) and at ex/em of $480 \mathrm{~nm} / 520 \mathrm{~nm}$ (bottom, in red; reduced).

Experiments were repeated 3 times. Student t-test, $\mathrm{SE}, \mathrm{N}=6,{ }^{*} \mathrm{P}<0.05, * * \mathrm{P}<0.01,{ }^{*} * \mathrm{P}<0.005$. Scale bar indicates $1 \mathrm{~cm}$. Abbreviations used: DCF, 2',7'-Dichlorofluorescein; ex/em, excitation/emission; L, local; S, systemic; roGFP1, reduction-oxidation sensitive green fluorescent protein 1; ROI, region of interest.

Figure 2. Whole-plant ratiometric fluorescence measurements of cytosolic roGFP1-expressing plants subjected to wounding

A. Representative images of whole-plant roGFP1 florescence (top; 420 and $480 \mathrm{~nm}$ excitations are in green and red, respectively), and statistical analysis of $420 \mathrm{~nm}, 480 \mathrm{~nm}$ and $420 \mathrm{~nm} / 480 \mathrm{~nm}$ ratio (bottom) of untreated cytosolic roGFP1 plants (Control), or cytosolic roGFP1 plants subjected to a 15 min fumigation with $5 \mathrm{mM}$ hydrogen peroxide $\left(\mathrm{H}_{2} \mathrm{O}_{2}\right)$ or $5 \mathrm{mM}$ Dithiothreitol (DTT). Ratios of florescence intensities obtained from the $\mathrm{H}_{2} \mathrm{O}_{2}$ and DTT treatments were used for range normalization of the degree of roGFP1 oxidation (similar to Schwarzländer et al., 2008) shown in B.

B. Representative time-lapse images (left) and line graphs of $420 / 480 \mathrm{~nm}$ ratio for the entire time course (ROls used to generate the graphs are shown in the images as dotted yellow ovals; middle), and bar graphs showing degree of roGFP1 oxidation for the 0 and 60 min time points calculated using the normalization range obtained in $\mathrm{A}$ (right), generated for the control untreated plants shown in Figure 1B.

C. Same as in B., but for the wounded plants shown in Figure 1B.

Experiments were repeated 3 times. Student t-test, $\mathrm{SE}, \mathrm{N}=6,{ }^{*} \mathrm{P}<0.05$. Scale bar indicates $1 \mathrm{~cm}$. Abbreviations used: DTT, Dithiothreitol; L, local; S, systemic; roGFP1, reduction-oxidation sensitive green fluorescent protein 1; ROI, region of interest. 


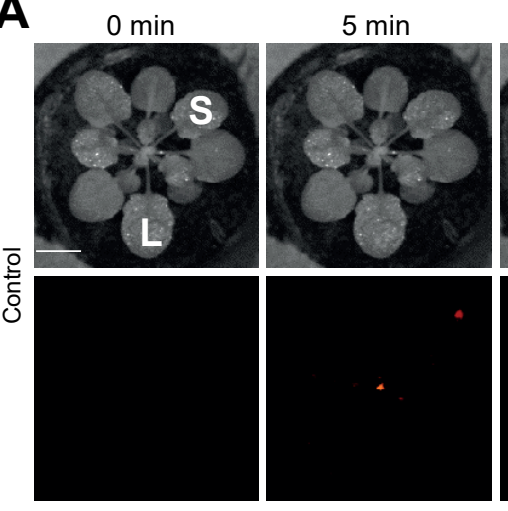

$0 \min$
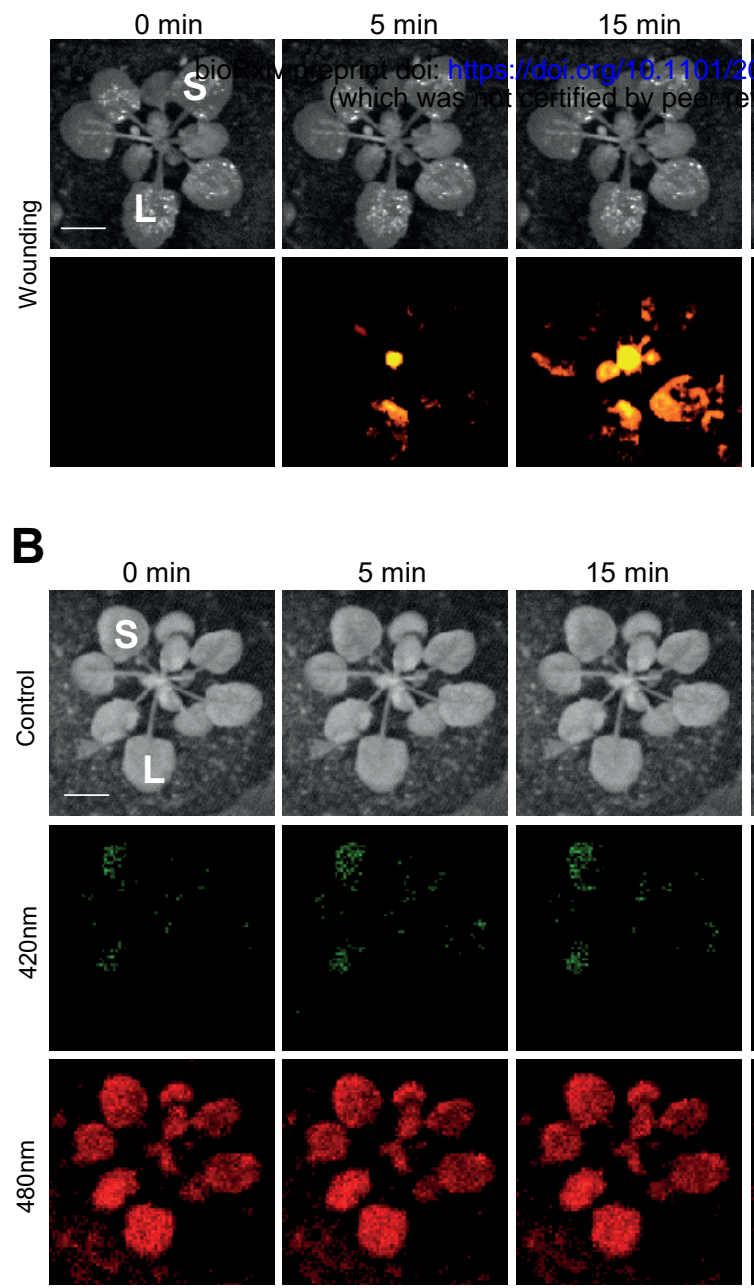

$0 \mathrm{~min}$

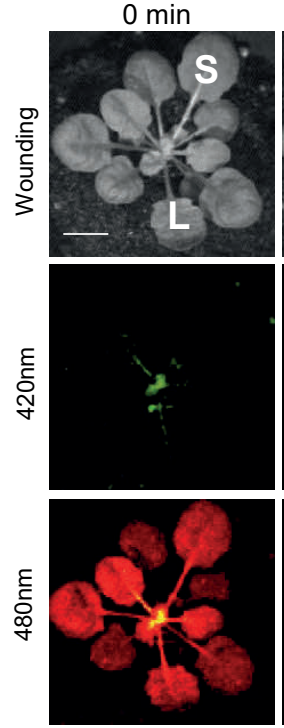

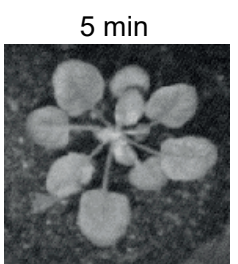
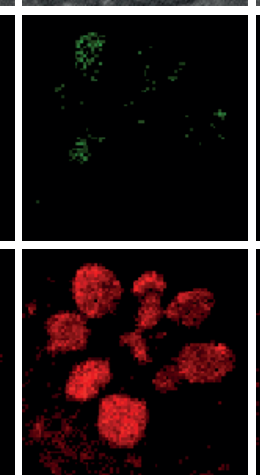

$5 \min$
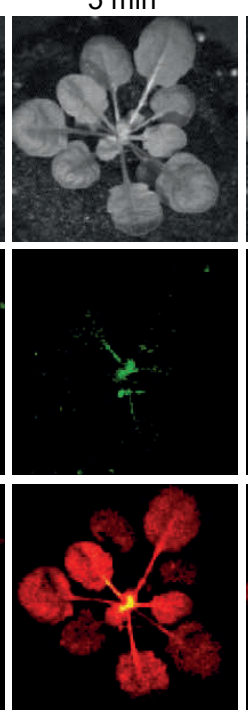

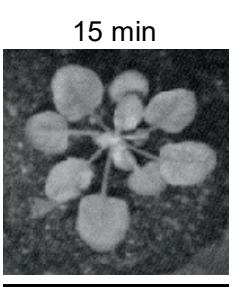

$15 \min$

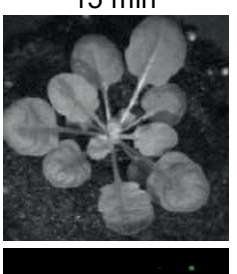

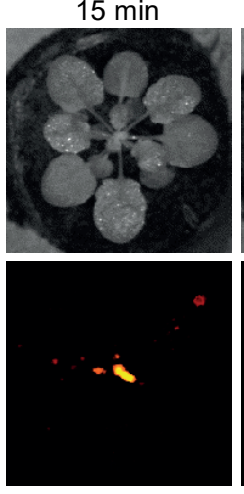

$15 \mathrm{~min}$

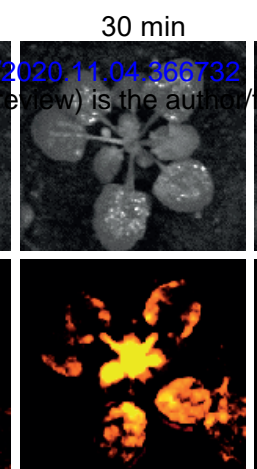

$30 \mathrm{~min}$

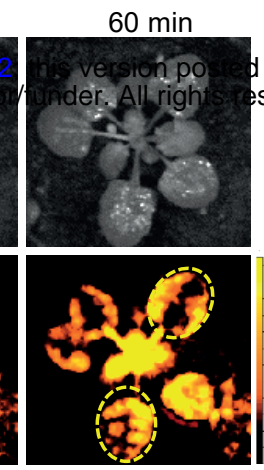

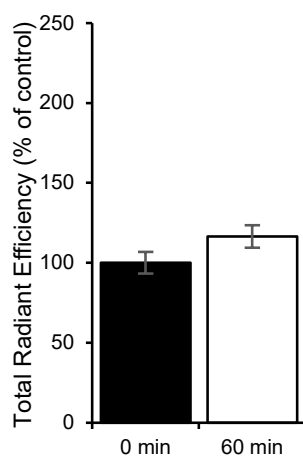

Time (min)

November 5, 2020. The côppyright holder for this prep No reuse allowed Without permission.**
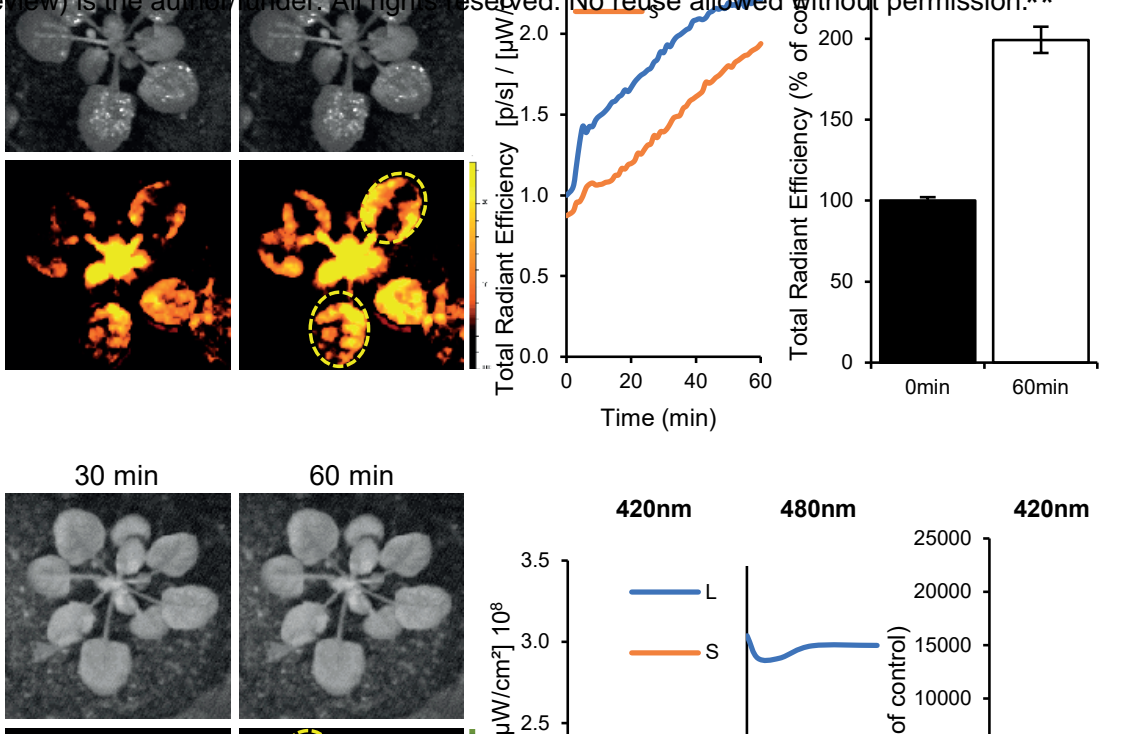

$60 \min$
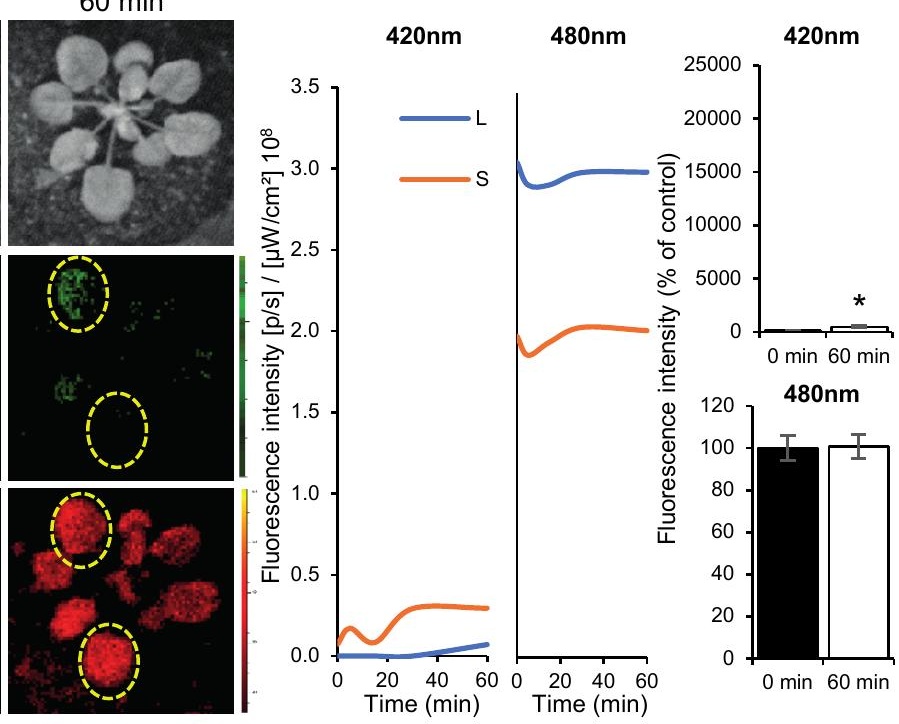

$30 \min$

$60 \min$
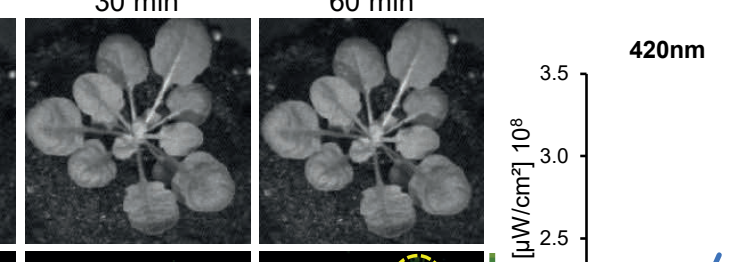

480nm

$420 \mathrm{~nm}$
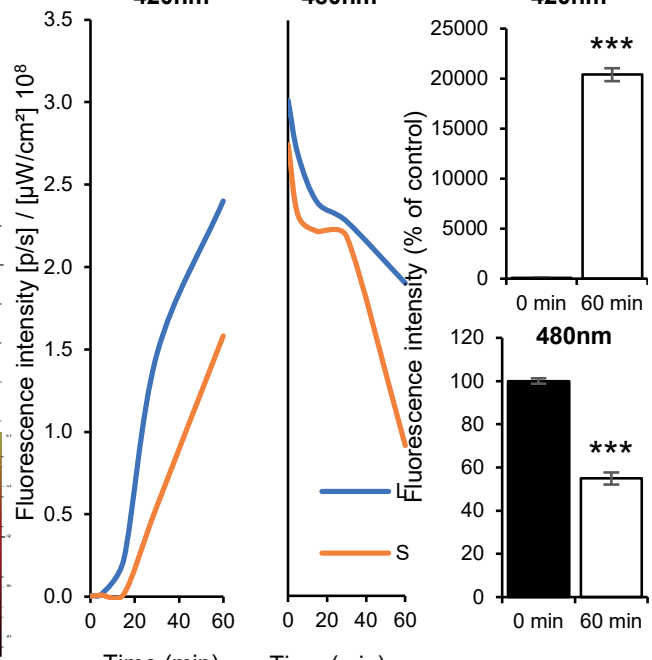

Figure 1. Imaging of ROS and redox levels in whole plants subjected to wounding

A. Representative time-lapse images of whole-plant ROS levels (indicated by DCF oxidation) in wild type Arabidopsis thaliana (Col-0) plants, untreated (control, top), or wounded (wounding, bottom; applied to leaf L only), are shown on left; representative line graphs showing continuous measurements of ROS levels in local (L) and systemic (S) leaves over the entire course of the experiment $(0$ to $60 \mathrm{~min}$ ) are shown at the middle (ROls used to generate the line graphs are indicated with dashed yellow ovals on left); and statistical analysis of ROS levels in local and systemic leaves at 0 and $60 \mathrm{~min}$ is shown on right.

B. Same as in A., but for transgenic plants overexpressing the roGFP1 protein at the cytosol (Jiang et al., 2006). Fluorescence was measured at excitation/emission (ex/em) of $420 \mathrm{~nm} / 520 \mathrm{~nm}$ for oxidized roGFP1 (middle, in green; oxidized) and at ex/em of $480 \mathrm{~nm} / 520 \mathrm{~nm}$ (bottom, in red; reduced).

Experiments were repeated 3 times. Student t-test, $\mathrm{SE}, \mathrm{N}=6,{ }^{*} \mathrm{P}<0.05,{ }^{*} \mathrm{P}<0.01,{ }^{*} * \mathrm{P}<0.005$. Scale bar indicates $1 \mathrm{~cm}$. Abbreviations used: DCF, 2',7'-Dichlorofluorescein; ex/em, excitation/emission; L, local; S, systemic; roGFP1, reduction-oxidation sensitive green fluorescent protein 1; ROI, region of interest 
